\title{
Size-frequency statistics of boulders on global surface of asteroid 25143 Itokawa
}

\author{
Tatsuhiro Michikami ${ }^{1}$, Akiko M. Nakamura ${ }^{2}$, Naru Hirata ${ }^{3}$, Robert W. Gaskell ${ }^{4}$, Ryosuke Nakamura ${ }^{5}$, \\ Takayuki Honda ${ }^{2}$, Chikatoshi Honda ${ }^{6}$, Kensuke Hiraoka ${ }^{2}$, Jun Saito ${ }^{7}$, Hirohide Demura ${ }^{3}$, \\ Masateru Ishiguro $^{8}$, and Hideaki Miyamoto ${ }^{9}$ \\ ${ }^{1}$ Fukushima National College of Technology, Iwaki, Fukushima 970-8034, Japan \\ ${ }^{2}$ Graduate School of Science, Kobe University, Kobe, Japan \\ ${ }^{3}$ University of Aizu, Aizu-Wakamatsu, Fukushima, Japan \\ ${ }^{4}$ Planetary Science Institute, Tucson, Arizona, USA \\ ${ }^{5}$ National Institute of Advanced Industrial Science and Technology (AIST), Tsukuba, Ibaraki, Japan \\ ${ }^{6}$ Institute of Space and Astronautical Science, Japanese Aerospace Exploration Agency, Kanagawa, Japan \\ ${ }^{7}$ PASCO Corporation, Meguro-ku, Tokyo, Japan \\ ${ }^{8}$ Seoul National University, Seoul, Korea \\ ${ }^{9}$ The University Museum, University of Tokyo, Tokyo, Japan
}

(Received February 18, 2007; Revised June 24, 2007; Accepted July 29, 2007; Online published February 12, 2008)

\begin{abstract}
The surface of asteroid 25143 Itokawa is covered with numerous boulders although gravity is very small compared with that of other asteroids previously observed from spacecraft. Here we report the size-frequency statistics of boulders on the entire surface of Itokawa based on high-resolution images ( 1 pixel $\sim 0.4 \mathrm{~m})$ obtained by the Hayabusa spacecraft. There are 373 boulders larger than $5 \mathrm{~m}$ in mean horizontal dimension on the entire surface $-0.393 \mathrm{~km}^{2}$ - and the number density is nearly $10^{3} / \mathrm{km}^{2}$. The cumulative boulder size distribution on the entire surface has a power-index of $-3.1 \pm 0.1$. For the east and west sides and the head and body portions of Itokawa, the power-index of the size distributions and the number densities of boulders of these areas are thought to be similar from the statistical point of view. A global mapping of boulders shows that there is no apparent correlation in the locations of boulders and craters. The ratio of the total volume of the boulders to the total excavated volume of the craters on Itokawa is $\sim 25 \%$ when only craters larger than $50 \mathrm{~m}$ in mean diameter are considered, and this ratio is extremely larger than that on Eros and the Moon, respectively. The origin of boulders on the surface of Itokawa was examined quantitatively by calculating the number of boulders and the size of the largest boulder using a model based on impact cratering experiments. The result indicated that the boulders on the surface of Itokawa cannot solely be the product of craters. Our results suggest that the boulders originated from the disruption of the larger parent body of Itokawa, as has been described in previous papers (Fujiwata et al., Science, 312, 1330-1334, 2006; Saito et al., Science, 312, 1341-1344, 2006).
\end{abstract}

Key words: Boulder, size distribution, Itokawa, asteroid.

\section{Introduction}

The existence of boulders on asteroids provides an opportunity to study the physical properties and the geological evolution of asteroid surfaces and, in particular, their collisional history. The boulders are formed by impact cratering and/or catastrophic disruption of the parent asteroid. The boulders on small bodies, such as the Martian satellites and the two asteroids 243 Ida and 433 Eros explored by previous space missions, are thought to be attributed to impact cratering (e.g., Lee et al., 1996; Thomas et al., 2001). During impact cratering, only the ejecta whose velocities are smaller than the escape velocity can fall back and settle on the surface under the influence of the gravity of asteroid. Before the arrival of the Hayabusa spacecraft to asteroid 25143 Itokawa, the number of meter-scale boulders (or, conversely, the extent of meter-scale flat ar-

Copyright (c) The Society of Geomagnetism and Earth, Planetary and Space Sciences (SGEPSS); The Seismological Society of Japan; The Volcanological Society of Japan; The Geodetic Society of Japan; The Japanese Society for Planetary Sciences; TERRAPUB eas) on the surface was a large concern from the engineering point of view in terms of successful sampling. Itokawa is an extremely small body (mean diameter $\sim 320 \mathrm{~m}$ ) compared with asteroids explored previously (mean diameter $\sim 12-50$ $\mathrm{km}$ ) and the escape velocity is very small (sub-meter per second); consequently, before the arrival of the Hayabusa spacecraft, most scientists thought that there would be only a few boulders on its surface. However, the images taken by the Asteroid Multi-band Imaging Camera (AMICA) on board the Hayabusa spacecraft showed that Itokawa is covered with a surprisingly large number of boulders (Fujiwara et al., 2006; Saito et al., 2006). Based on the appearance of Itokawa's surface and the sizes of the boulders, Fujiwara et al. (2006) and Saito et al. (2006) suggested that such numerous boulders were produced when Itokawa was generated by a catastrophic disruption of its parent body.

The aim of the investigation reported here was to examine quantitatively the origin of boulders on the surface of Itokawa. To this end, we investigated the spatial distribution and the size distribution of the numerous boulders on 


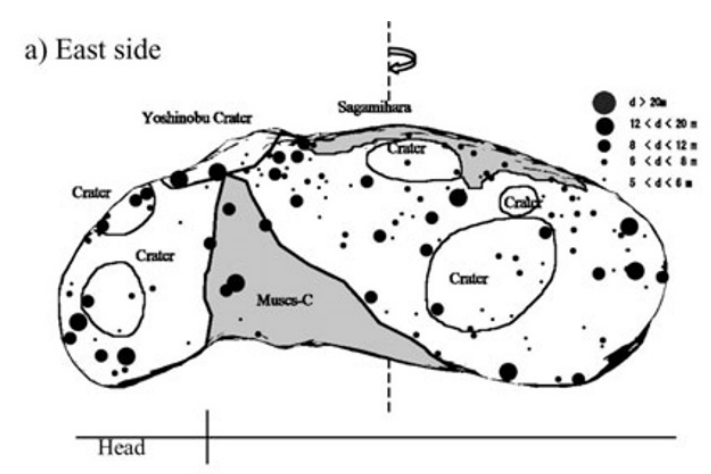

b) West side

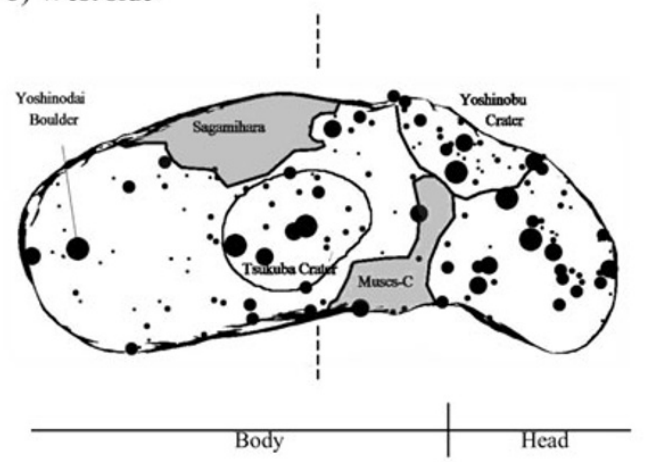

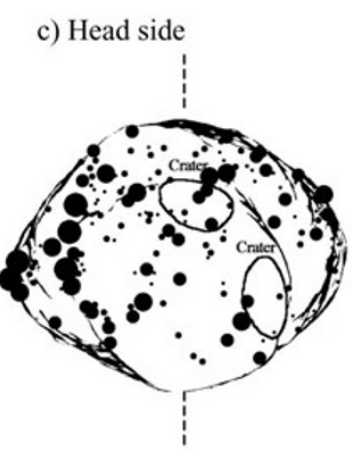

d) Body side

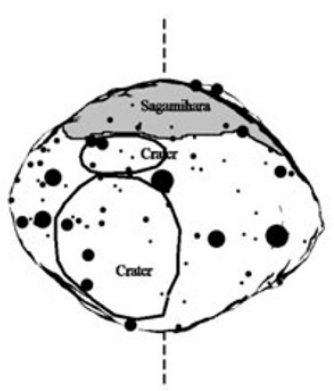

Fig. 1(a). Maps of boulders larger than $5 \mathrm{~m}$ in the mean horizontal dimensions on Itokawa. Circular points are boulders, solid lines are boundaries of rough/smooth terrains, outline of Itokawa and crater candidates larger than $50 \mathrm{~m}$ in mean diameter, dashed lines are the spin axis of Itokawa, shadows show the smooth terrains, Muses-C and Sagamihara. Maps (a, b, c, and d) of boulders on Itokawa are viewed from the east, west, head and body sides, respectively. We used the shape model constructed by Gaskell et al. (2006) to determine the positions of the boulders and the shape of Itokawa. Data on boulders were collected from eight images (ST2473604354, ST2481211873, ST2482160259, ST2484352917, ST2485860275, ST2492225173, ST2492513077, and ST2493031594) taken at an altitude between 3.779 and $4.913 \mathrm{~km}$. From one of these, the mean horizontal dimension of a boulder is defined as the additional mean size of the dimensions of the two axes, which represent the longest dimension of the boulder and then the longest dimension perpendicular to this.

the west and east sides and on the head and body portions based on images taken by the Hayabusa spacecraft and compared the observed number of boulders with the calculated value using a model based on impact cratering experiments.

\section{Methodology}

In order to investigate the size-frequency statistics of the numerous boulders on Itokawa, we have measured the mean horizontal dimensions of positive relief features larger than $5 \mathrm{~m}$ over the entire surface. We call these larger $(\geq 5 \mathrm{~m})$, positive relief features "boulders". This critical value is not hampered by the limited spatial resolution on the entire asteroid imaged by the spacecraft. On Eros, Thomas et al. (2001) used the mean horizontal dimensions as the boulder size (they mapped boulders larger than $15 \mathrm{~m}$ over the entire surface). In this study, we also used the mean horizontal dimension as the boulder size in order to be able to compare our data with the Eros data of Thomas et al. (2001). Our first step was to measure the longest dimension of the boulder and the longest dimension perpendicular to this. We defined the mean horizontal dimension of a boulder as the additional mean size of these dimensions of these two axes. Measurements were made in various areas on Itokawa from eight images (resolutions $\sim 0.4 \mathrm{~m} /$ pixel) acquired between 19 and 26 October 2005. We confirmed the positive relief features by comparing these images with stereo coverage acquired from the nominal hovering position (Home posi- tion) at an altitude of about $7 \mathrm{~km}$.

We have observational biases on their global mapping. Boulder mapping is affected by variations in spatial resolution and in the availability of stereo coverage. The regions at the middle and low latitudes were viewed on several images at a resolution $\sim 0.4 \mathrm{~m} / \mathrm{pixel}$ and with substantial stereo coverage. In contrast, the regions at the high latitude were viewed only on a few images at a resolution of $\sim 0.4 \mathrm{~m} / \mathrm{pixel}$ and with no stereo coverage. Therefore, it is possible that several boulders located at the high-latitude locations were not counted in our data.

The surface of Itokawa is separated into "rough terrain", which occupies about $80 \%$ of Itokawa's surface with numerous boulders, and "smooth terrain" covered by finer particles. A surprising number of positive relief features appear on the rough terrain. Isolated positive relief features might include partially buried boulders, weathered boulders with subdued outlines, piles of regolithic scree, protruding bedrocks, raised crater rims, and intersecting crater walls. In this study, the boulder counts might contain some of these.

\section{Observation Results}

\subsection{Spatial distribution and size of boulders}

The spatial distribution of boulders is shown in Fig. 1 (a, b). The number of large boulders - more than $20 \mathrm{~m}$ - is six on Itokawa. The largest boulder is called "Yoshinodai", 
a) East side

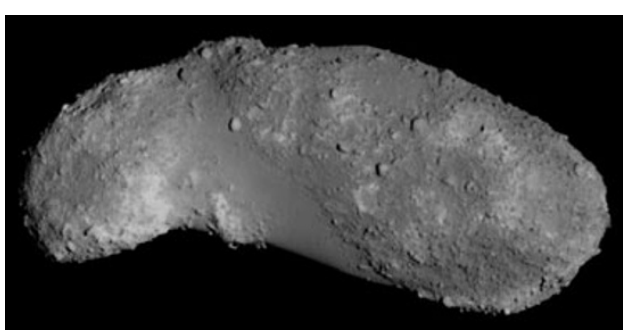

b) West side

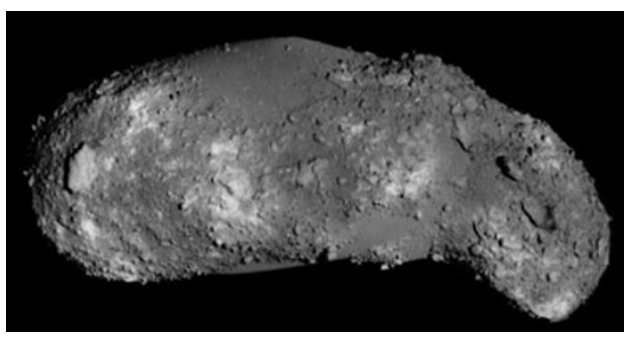

c) Head side

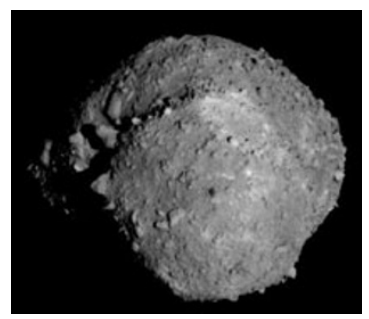

d) Body side

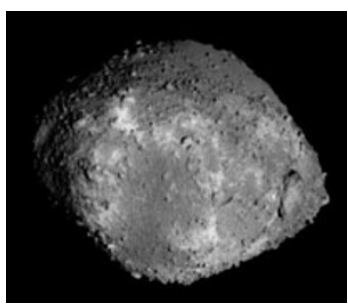

Fig. 1(b). Images of Itokawa taken from a nominal hovering position (Home position) at an altitude of about $7 \mathrm{~km}$. Itokawa's orthogonal axes are 535, 294, and $209 \mathrm{~m}$ (Fujiwara et al., 2006). Images (a, b, c, and d) on Itokawa are viewed from the east, west, head and body sides, respectively. Image ID's: ST2417520833, ST2421011334, ST2423460670, and ST2424157005.

with dimensions of $\sim 50 \times 30 \times 20 \mathrm{~m}$ (Saito et al., 2006). Some of these large boulders are taller than they are wide, which is a rather unstable orientation. There are 373 boulders larger than $5 \mathrm{~m}$ in the mean horizontal dimension on the entire surface $\left(0.393 \mathrm{~km}^{2}\right.$; Demura et al., 2006) of Itokawa. Note that there are more than 500 boulders on Itokawa when boulder size is defined as the maximum size of boulders (Saito et al., 2006). The difference between these two numbers is due to the difference in the definition of boulder size. For an identical boulder, the mean horizontal dimension is smaller than the maximum one. Thus, the number of boulders determined on the basis of the mean horizontal dimensions is smaller than that based on the maximum ones. The average number density of boulders larger than $5 \mathrm{~m}$ is nearly $10^{3} / \mathrm{km}^{2}$, which is slightly larger than that on Eros (Thomas et al., 2001).

The cumulative boulder size distribution per unit area on the entire surface of Itokawa has a power-index of $-3.1 \pm$ 0.1 (Fig. 2), which is similar to those found on other airless objects. The power-index for lunar boulders ranges from -1.8 to -3.7 (Cintala et al., 1982; Cintala and McBride, 1995), with sizes ranging from a few meters to tens of meters. The global data of Eros have a power-index of -3.2 between sizes of 15 and $80 \mathrm{~m}$ (Thomas et al., 2001). Boulders on the Martian satellite Phobos also give a powerindex of -3.2 (Thomas et al., 2000). Note that the slope of the boulder size distribution on Itokawa becomes less steep (-2.8) (Saito et al., 2006) when boulder size is defined as the maximum size of the boulders. In general, the powerindex of the size distributions is affected by the details of the binning and the range in size (Colwell, 1993). Moreover, several of Itokawa's boulders are larger than $20 \mathrm{~m}$, and their shapes are more elongated when compared to the smaller ones. Therefore, the slope of the boulder size distribution with the boulder's size defined as the average horizontal one is steeper than that defined as the maximum one.

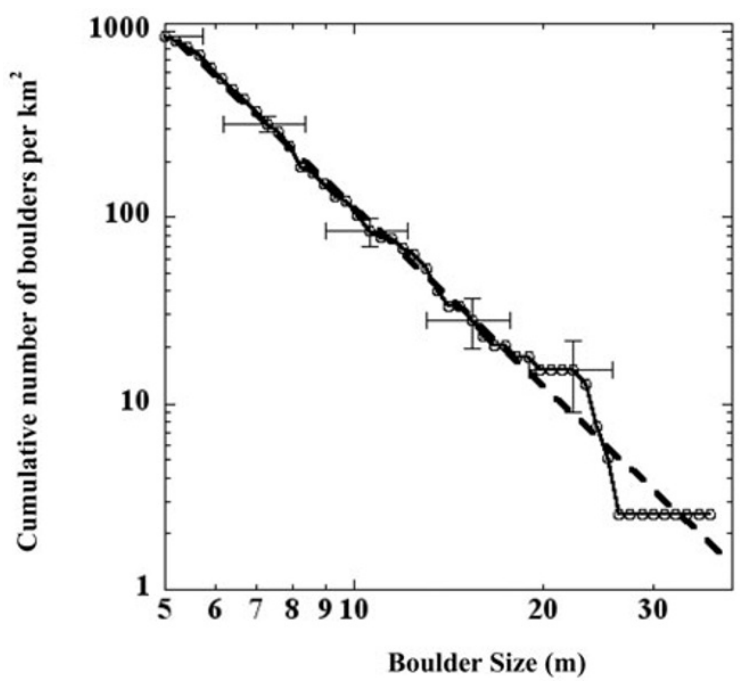

Fig. 2. Cumulative boulder size distribution per unit area on the entire surface of Itokawa. The horizontal axis shows the size of the boulder, where the size is defined as the mean horizontal dimensions of the boulder. The vertical axis shows the number of boulders larger than a particular size per unit area. Horizontal error bars indicate the error caused by the scatter among different measurements performed by three people, and is within a tolerance of $15 \%$ for the size. Vertical error bars indicate the root of the cumulative number of counting boulders divided by the total area of Itokawa. The error bars are given only for five points to make figure legible. The broken line is a fitted regression line to the data, and the power-law index of size distribution is $-3.1 \pm 0.1$, which is similar to those found on other airless objects (see text).

The spatial densities of boulders at different locations on Itokawa are not the same, which is also true for Eros, with the rough and smooth terrains differing by several factors or a magnitude in terms of the number density of boulders. The smooth terrains (Muses-C and Sagamihara) of Itokawa appear to lack boulders, which we will discuss in more detail in the following subsection. The background 


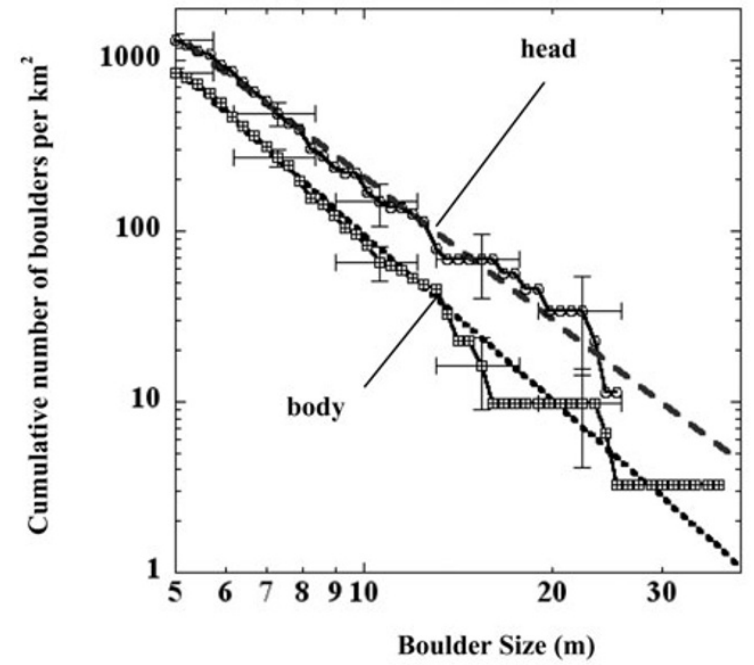

Fig. 3. Cumulative boulder size distribution per unit area on head and body portions of Itokawa. The broken and dotted lines are the regression lines for the data of the head and body, respectively. The slope (the power-index $-2.8 \pm 0.1$ ) of boulder size distribution on the head is not as steep as the slope (the power-index $-3.2 \pm 0.1$ ) of that on the body; however, the calculation of rank-sum test shows that the difference was not statistically significant at the 0.05 probability level. The head and body also differ in the number density of boulders, with that $\left(1.3 \times 10^{3} / \mathrm{km}^{2}\right)$ on the head being 1.5 -fold larger than that on the body $\left(8.5 \times 10^{2} / \mathrm{km}^{2}\right)$.

topography of the rough terrain is distinctly rougher than that of the smooth terrain, making isolated boulders more difficult to identify.

The shape of Itokawa resembles a sea otter-with a smaller, rather round head and a larger oval body mass. We also investigated the statistics of boulders in two portions of Itokawa, the otter's head and the body. The boulder size distributions of the head and body are shown in Fig. 3. The slope (power-index $-2.8 \pm 0.1$ ) of boulder size distribution on the head is not as steep as the slope (power-index $-3.2 \pm 0.1$ ) of that on the body. Although the head portion does not contain smooth terrains while the body portion does, the power-index on the body does not change even if the smooth terrains are not taken into account. The difference in the power-index appears to be due to the characteristic topography of the head and body portions of Itokawa. However, the calculation of the rank-sum test shows that this difference is not statistically significant at the 0.05 probability level. The head and body also appear to differ in the number density of boulders-with that for the head $\left(1.3 \times 10^{3} / \mathrm{km}^{2}\right)$ being 1.5-fold higher than that for the body $\left(8.5 \times 10^{2} / \mathrm{km}^{2}\right)$. However, the ratio is reduced to 1.3 when only the rough terrains are taken into account for the body.

Figure 4 shows that there are several large $(>20 \mathrm{~m})$ boulders on the west side, while there are few large boulders on the east side. The slope (power-index $-2.9 \pm 0.1$ ) of the size distribution of boulders larger than $5 \mathrm{~m}$ on the west side is not as steep as the slope (power-index $-3.2 \pm 0.1$ ) of that on the east side. This is caused by the existence of larger boulders on the west side. A possible scenario for the existence of large boulders on the west side is that

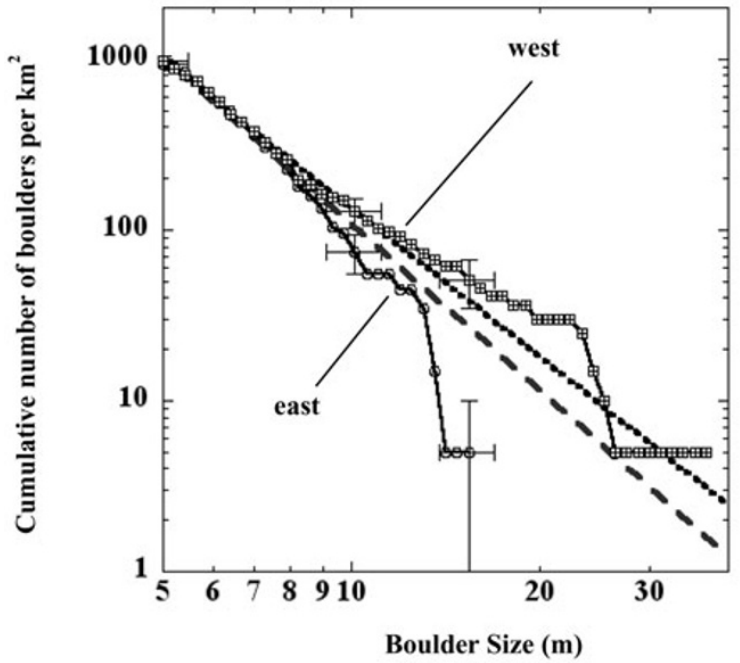

Fig. 4. Cumulative boulder size distribution per unit area on the east and west sides of Itokawa. The broken and dotted lines are the regression lines for the data of the east and west, respectively. The slope (power-index $-2.9 \pm 0.1$ ) of the size distribution on the west side is not as steep as the slope (power-index $-3.2 \pm 0.1$ ) of that on the east side because there are several large $(>20 \mathrm{~m})$ boulders on the west side while there are few large boulders on the east side. However, the calculation of rank-sum test shows that the difference was not statistically significant at the 0.05 probability level. The number density of boulders on the east side is roughly the same as that on the west, which is similar to that on the entire surface.

large boulders, which are formed by impact cratering and/or catastrophic disruption of the parent body, spend a time in temporary orbit around Itokawa before settling on the west side of the rotational leading surfaces. This mechanism, such as the asymmetric rotational sweep-up of launched fragments, was proposed by Geissler et al. (1994, 1996a, 1996b). Geissler et al. (1994, 1996a) noted that the portions of the eastern and western regions of asteroid Ida where most blocks are found coincide with the asteroid's leading sides in a rotational sense. However, we suggest that this mechanism does not work efficiently for most of boulders on Itokawa: the number density of boulders on the east side is roughly the same as that on the west; therefore, most of boulders do not migrate to the west side by the mechanism in a rotational sense. A detailed search is required to detect the effect of the rotation on large boulder emplacement.

\subsection{Relation between craters and boulders}

We found that boulders and other small overhang features dominate the landscape of Itokawa and that craters are less obvious when compared with those on other asteroids observed by spacecrafts. There are nine crater candidates with a mean diameter $>50 \mathrm{~m}$ and few small craters with a mean diameter $<10 \mathrm{~m}$ (Hirata et al., 2007). The boulders on Itokawa appear to exist regardless of the position of the craters. In general, however, boulders on other small bodies observed by spacecraft show some relationship with the positions of the large craters. On the Martian satellite Phobos, nearly all boulders are located close to large impact craters, and most large boulders are associated with the Stickney crater (Thomas, 1979). On Deimos, Lee et al. (1986) argued — on the basis of the proximity and radial symmetry of the distribution of the largest boulders-that 
the latter may have originated from the Voltaire crater. The boulders on Ida lie within or near the rims of the Lascaux and Mammoth craters (Lee et al., 1996). Thomas et al. (2001) suggest that most large boulders on Eros originate from a relatively young Shoemaker crater based on the images obtained by spacecraft and their ejecta-emplacement model.

One plausible process explaining the mismatching of boulders with the craters on Itokawa is as follows. Most of the boulders are surviving fragments from the parent body of Itokawa, accreted after its breakup (Fujiwara et al., 2006). Impact ejecta (including boulders, cobbles, and pebbles) were uniformly spread over the entire surface; however, cobbles and pebbles were reallocated after their accumulation by global vibration (seismic shaking) caused by impact cratering, among others. In other words, finer particles, such as cobbles and pebbles, migrated into the smooth terrains, which are areas of potential gravitational plus rotational lows. This would occur because finer particles have a higher mobility due to their lower friction angle. Conversely, the boulders were stranded at the surface due to their higher friction angle, subsequently forming rough terrains. These mechanisms are indicated by Miyamoto et al. (2007) as granular processes (granular processes become major resurfacing processes because of Itokawa's small size). The boulders on the smooth terrains are expected to be covered with finer particles having a high mobility (as a result, the number of boulders on the smooth terrains is very small compared with that on the rough terrains). The boulders on the rough terrains of Itokawa would hold the original positions and exist regardless of the craters. In the next section, we describe the quantitative estimate of the total volume of craters and boulders in order to investigate the origin of Itokawa.

Another characteristic of Itokawa is that there are extremely large boulders relative to the size of the largest craters, as has been well described by Fujiwara et al. (2006). Empirical relations between the largest boulder size $(L)$ and the source crater diameter $(D)$ have been developed for the Moon, Phobos, Deimos, and Ida (Lee et al., 1996). One of the relations is given by $L \sim 0.25 D^{0.7}$, with $L$ and $D$ expressed in meters (although this relation is tuned for craters $10^{3} \mathrm{~m} \leq D \leq 2 \times 10^{4} \mathrm{~m}$, it was originally synthesized from two other empirical relations applicable for even smaller craters on the Moon and the Earth and, therefore, can be applied for the largest crater candidate on Itokawa). On Eros, the size of the maximum boulder (130 $\mathrm{m}$ across) is also consistent with the prediction based on the empirical scaling laws. On Itokawa, the size of the largest crater candidate is about $134 \mathrm{~m}$ in diameter (Hirata et al., 2007). The empirical equation predicts that the largest boulder on Itokawa is $7.7 \mathrm{~m}$ across, which is much smaller than the size of the largest candidate (Yoshinodai boulder $\sim 40 \mathrm{~m}$ in mean diameter) (Saito et al., 2006). Thus, the maximum size of the observed boulders on Itokawa is very large compared with those of boulders found on other airless bodies. This is thought to be evidence that most boulders are surviving fragments from the parent body of Itokawa, accreted after its breakup.

\section{Comparison with a Model}

In order to examine quantitatively the origin of numerous boulders on the surface of Itokawa - and not only the largest boulders, as discussed in the previous section-we first estimate the total volume of ejecta represented by the boulders and the total volume of ejecta by the craters on Itokawa. The ratio of boulder volume to crater volume enables the various hypotheses on the origin of the boulders to be tested. We next calculate the number of boulders by a model based on impact cratering experiments (Michikami et al., 2005; Michikami et al., 2007) and compare the observed number of boulders with the calculated values. Finally, we discuss if the boulders are mainly formed during impact cratering or during catastrophic disruption: the origin of boulders.

Nine craters larger than $50 \mathrm{~m}$ in mean diameter, including crater candidates, are found on Itokawa, as mentioned by Hirata et al. (2007). The volume of material removed in the formation of these craters can be estimated to $\sim 3.3 \times 10^{5} \mathrm{~m}^{3}$, if the excavated volume is half that of the crater volume (this method is the same as that of Thomas et al. (2001); namely, the crater volume, $V$, is given by $V \sim 0.07 D^{3}$, where $D$ is apparent crater diameters, and the excavated volume is the half). This estimate does not include the contribution from craters smaller than $50 \mathrm{~m}$ in diameter, but it nevertheless accounts for most of the ejecta produced because of the steepness of the power dependence of ejecta volume on crater size. Table 1 shows the diameter of nine largest craters, the estimated total volume, and the mass ejected from each crater. The two largest crater candidates-with a diameter of $134 \mathrm{~m}$ and $128 \mathrm{~m}$, respectively-contributed $\sim 1.6 \times 10^{5} \mathrm{~m}^{3}$ of ejecta, about half of all the excavated material. The total calculated volume of observed boulders (with the mean horizontal dimensions ranging from 5 to $40 \mathrm{~m}$ ) is $8.2 \times 10^{4} \mathrm{~m}^{3}$, assuming the heights are half the mean horizontal dimension. The boulders therefore account for $\sim 25 \%$ the excavated volume of these craters. This percentage is very much larger than that on Eros (less than 1\%) and even that on the Moon ( 5\%) (Thomas et al., 2001; Cintala et al., 1982). Most of the ejecta must have been lost owing to Itokawa's low escape velocity. Thus, the high percentage of the total volume of the boulders to the total excavated volume of the craters on Itokawa implies that boulders cannot solely be produced as products of cratering on the surface of Itokawa.

In order to investigate quantitatively whether the numerous boulders were formed only during impact cratering especially when considering the escaping fraction, we calculate the number of boulders using a model based on impact cratering experiments. We assume that (1) the observed nine largest crater candidates (Hirata et al., 2007) are the source craters for the ejecta boulders, (2) the ejecta mass distribution during one impact cratering is given by a power-law distribution based on the results of the impact experiments, and (3) the ejecta having a velocity less than the escape velocity of Itokawa can fall back onto the surface as boulders.

The constant power-index of $-2 / 3$ for the cumulative ejecta mass distribution was found in previous laboratory cratering experiments of porous sintered glass beads targets (Michikami et al., 2005). A similar constant exponent was 
Table 1. The diameter $(D)$ of nine craters larger than $50 \mathrm{~m}$, the excavated volume $(0.5 \times V)$ from the crater, and the estimated total mass $\left(M_{\text {total }}\right)$ ejected from the crater, where $V$ is given by $V \sim 0.07 D^{3}$ and $M_{\text {total }}$ are given by $0.5 \rho V$. The bulk density $\rho$ of Itokawa is $1950 \mathrm{~kg} \mathrm{~m}^{-3}$ (Abe $e t$ al., 2006). The data of these crater diameters are quoted from Hirata $e t$ al. (2007).

\begin{tabular}{ccc}
\hline$D[\mathrm{~m}]$ & $0.5 \times V\left[\mathrm{~m}^{3}\right]$ & $M_{\text {total }}[\mathrm{kg}]$ \\
\hline 134 & $8.4 \times 10^{4}$ & $1.6 \times 10^{8}$ \\
128 & $7.3 \times 10^{4}$ & $1.4 \times 10^{8}$ \\
117 & $5.6 \times 10^{4}$ & $1.1 \times 10^{8}$ \\
104 & $3.9 \times 10^{4}$ & $0.8 \times 10^{8}$ \\
102 & $3.7 \times 10^{4}$ & $0.7 \times 10^{8}$ \\
74 & $1.4 \times 10^{4}$ & $0.3 \times 10^{8}$ \\
64 & $0.9 \times 10^{4}$ & $0.2 \times 10^{8}$ \\
61 & $0.8 \times 10^{4}$ & $0.2 \times 10^{8}$ \\
54 & $0.6 \times 10^{4}$ & $0.1 \times 10^{8}$ \\
\hline (total) & $3.3 \times 10^{5}$ & $6.4 \times 10^{8}$ \\
\hline
\end{tabular}

found for ejecta from the Tycho crater on the moon, based on analyses of the spatial and size distribution of secondary craters (Hirata and Nakamura, 2006). According to Hirata and Nakamura (2006), the power-index of the ejecta mass distributions is not only close to that of other natural craters, but it is also close to that of boulders on the asteroid Eros. Thus, since the power-index is roughly the same regardless of the very different conditions, the relative ejecta size distributions would be independent of the ejection velocity, the material strength, the porosity and the size of source crater producing the ejecta. The mass distribution of ejecta having a velocity less than a given ejection velocity $(v)$ is given by a power distribution,

$$
N(>m,<v)=C(<v) m^{-2 / 3},
$$

where $m$ is the individual ejecta mass and $N$ is the cumulative number of ejecta or particle having a mass larger than $m$. The coefficient $C$ in Eq. (1) can be determined when the mass $\left(m_{l}\right)$ of the largest boulder fallen back to the surface is given for a single impact cratering; the cumulative number of boulders having a mass larger than $m_{l}$ is unity,

$$
N\left(>m_{l},<v_{e}\right)=1=C\left(<v_{e}\right) m_{l}^{-2 / 3} .
$$

Then we have

$$
C\left(<v_{e}\right)=m_{l}^{2 / 3},
$$

where $v_{e}$ is the escape velocity of Itokawa and $N\left(>m_{l},<\right.$ $v_{e}$ ) is the cumulative number of ejecta having a mass larger than $m_{l}$. The mass of the largest boulder, on the other hand, is derived from the following equation,

$$
\int_{0}^{m_{l}} n\left(m,<v_{e}\right) m d m=f_{e} M_{\text {total }},
$$

where $n\left(m,<v_{e}\right)$ is the number of ejecta with a mass varying from $m$ to $m+d m$ and with a velocity less than $v_{e}$. $f_{e}$ then indicates the mass fraction of the deposited ejecta mass to the total ejecta mass $\left(M_{\text {total }}\right)$ at one impact cratering event. From Eq. (1) and Eq. (2'), we obtain

$$
n\left(m,<v_{e}\right)=\frac{2}{3} m_{l}^{\frac{2}{3}} m^{-\frac{5}{3}} .
$$

As a result, the left hand side of Eq. (3) is given by

$$
\int_{0}^{m_{l}} n\left(m,<v_{e}\right) m d m=2 m_{l}
$$

Therefore, we obtain

$$
m_{l}=\frac{1}{2} f_{e} M_{\text {total }} \cdot
$$

The mass fraction $\left(f_{e}\right)$ of the ejecta having a velocity less than a given ejection velocity $(v)$ is expected to be a function of the ejection velocity and of the material strength and the bulk density of the surface in the strength regime of the cratering process (Housen et al., 1983). Laboratory cratering experiments revealed that cratering is mainly dependent upon the material strength of the surface, and not so greatly on the porosity (Housen, 1992; Michikami et al., 2007). In this paper, the material strength is changed within a range of plausible values in order to evaluate the effect on the fraction of the deposited boulder volume to total boulder volume at one impact cratering event. The material strength of Itokawa is considered to be less than $10 \mathrm{MPa}$ as follows. The bulk density of Itokawa is $\sim 1950 \mathrm{~kg} \mathrm{~m}^{-3}$ (Abe et al., 2006), which is less than that $\left(\sim 2600 \mathrm{~kg} \mathrm{~m}^{-3}\right)$ of the other S-type asteroids previously explored, Ida and Eros. The smaller bulk density indicates that Itokawa has a large bulk porosity of $\sim 40 \%$ (Abe et al., 2006) in comparison to that of S-type asteroids Ida and Eros, which have bulk porosities of $20 \%$ (Britt et al., 2002). The material strength of Eros was estimated to be $\sim 10 \mathrm{MPa}$ (Dombard and Freed, 2001). In previous impact experiments (Michikami et al., 2007), the material strength of the targets having a bulk porosity of $\sim 40 \%$ ranged from 0.5 to $5.5 \mathrm{MPa}$, and a number of ejecta with very low velocities (less than Itokawa's escape velocity) were observed. A weak surface combined with material strength of less than $0.1 \mathrm{MPa}$ would produce only a small amount of ejecta due to compaction (Housen and Holsapple, 2003). Thus, we do not use a value of less than $0.1 \mathrm{MPa}$ as the material strength in this paper.

According to results of laboratory impact cratering experiments (Michikami, 2001; Michikami et al., 2007), the value of $f_{e}$ is calculated by

$$
f_{e}=1-F\left(>v \sqrt{\frac{\rho}{Y}}\right),
$$

where $F$ is the mass fraction of the ejecta having a velocity larger than a given non-dimensional velocity $v \sqrt{(\rho / Y)}$ (ejecta velocity $(v)$, target density $(\rho)$ and material strength $(Y))$. When the non-dimensional scaling parameter $v \sqrt{(\rho / Y)}$ is smaller than $0.7, F$ is

$$
\begin{aligned}
F\left(>v \sqrt{\frac{\rho}{Y}}\right)= & 1-1.54\left(v \sqrt{\frac{\rho}{Y}}\right)+0.43\left(v \sqrt{\frac{\rho}{Y}}\right)^{2} \\
& +0.36\left(v \sqrt{\frac{\rho}{Y}}\right)^{3}
\end{aligned}
$$

When the non-dimensional scaling parameter $v \sqrt{(\rho / Y)}$ is larger than $0.7, F$ is

$$
F\left(>v \sqrt{\frac{\rho}{Y}}\right)=0.142\left(v \sqrt{\frac{\rho}{Y}}\right)^{-1.65} .
$$


Table 2. Estimated largest boulder size and number of boulders larger than $5 \mathrm{~m}$.

\begin{tabular}{lccc}
\hline Material strength & $10 \mathrm{MPa}$ & $1 \mathrm{MPa}$ & $0.1 \mathrm{MPa}$ \\
\hline $\begin{array}{l}\text { Mass fraction }\left(f_{e}\right) \text { of } \\
\text { ejecta fallen back to } \\
\text { the surface }\end{array}$ & $0.4 \%$ & $1.4 \%$ & $4.3 \%$ \\
\hline $\begin{array}{l}\text { Diameter }\left(D_{\max }\right) \text { of the } \\
\text { largest boulder from } \\
\text { the largest crater }\end{array}$ & $9 \mathrm{~m}$ & $13 \mathrm{~m}$ & $19 \mathrm{~m}$ \\
\hline $\begin{array}{l}\text { Estimated number of } \\
\text { boulders larger than } 5 \mathrm{~m}\end{array}$ & 14 & 33 & 70 \\
\hline
\end{tabular}

When the results of laboratory experiments are applied to an asteroid, the ejecta velocity, the bulk density, and the material strength are interpreted as the escape velocity, the bulk density and the material strength of the asteroid, respectively. In the case of Itokawa, the escape velocity is $0.20 \mathrm{~m} / \mathrm{s}$, and the bulk density is $1950 \mathrm{~kg} \mathrm{~m}^{-3}$. The material strengths are thought to range from 0.1 to $10 \mathrm{MPa}$, as mentioned before. The values of $f_{e}$ on Itokwa are 0.4 , 1.4 and $4.3 \%$ when the material strengths are 10, 1 and $0.1 \mathrm{MPa}$, respectively.

From Eq. (2') and Eq. (6), Eq. (1) can be rewritten as

$$
N\left(>m,<v_{e}\right)=\left(\frac{1}{2} f_{e} M_{\text {total }}\right)^{\frac{2}{3}} m^{-\frac{2}{3}} .
$$

The number of boulders larger than $5 \mathrm{~m}$ produced from one crater can be calculated from Eq. (10) when $m$ is the mass of boulder having a mean horizontal diameter of $5 \mathrm{~m}$ and it is assumed that the height is half of that. The value of $m$ is then $6.4 \times 10^{4} \mathrm{~kg}\left(=2 / 3 \pi r^{3} \rho\right.$, where $r$ is $2.5 \mathrm{~m}$, the mean horizontal radius of boulder, and $\rho$ is $1950 \mathrm{~kg} \mathrm{~m}^{-3}$, the bulk density of Itokawa). The total number of boulders larger than $5 \mathrm{~m}$ on the entire surface of Itokawa can be estimated by the sum of the number of the boulders produced from each crater.

The mass of the largest boulder produced from the largest crater (diameter $134 \mathrm{~m}$ ) can be estimated from Eq. (6) when $M_{\text {total }}$ is the total ejecta mass from that crater. The diameter $\left(D_{\max }\right)$ of the largest boulder from that crater diameter is then given by

$$
D_{\max }=\left(\frac{6 f_{e} M_{\text {total }}}{\pi \rho}\right)^{\frac{1}{3}} .
$$

Table 2 shows the estimated total number of boulders larger than $5 \mathrm{~m}$ on the entire surface of Itokawa and the diameter $\left(D_{\max }\right)$ of the largest boulder from the largest crater (diameter $134 \mathrm{~m}$ ). Table 2 shows that the number of boulders increases with decreasing material strength, with the reason for this being that the mean velocity of ejecta decreases with decreasing material strength. In this model, the size of the largest boulder also slightly increases with decreasing material strength. Such a tendency was found in some of the experiments of one of authors (Michikami et al., 2007), whereas a model of impact spallation predicts complicated material dependency of the thickness of spall fragments (Melosh, 1984).
The estimated number of boulders is smaller than observed data by nearly one order of magnitude, and the calculated size of the largest boulder is smaller than the observed size. These results probably indicate that it is difficult to produce as many boulders on the surface as were observed when only impact cratering is considered as the source; it is therefore likely that most boulders are surviving fragments from the parent body of Itokawa, accreted after its breakup. Fujiwara et al. (2006) suggested that Itowaka is a rubble-pile body because of its low bulk density, high porosity, boulder-rich appearance, and shape and that the large boulders are surviving fragments from the parent body of Itokawa. Our results support this idea quantitatively from the estimated number of boulders and the calculated size of the largest boulder by a model based on impact cratering experiments.

\section{Conclusion}

In the high-resolution images of asteroid 25143 Itokawa, we have identified 373 boulders larger than $5 \mathrm{~m}$ in mean horizontal dimension on the entire surface $\left(0.393 \mathrm{~km}^{2}\right)$ of the asteroid and found that the number density is nearly $10^{3} / \mathrm{km}^{2}$. This value is slightly larger than that determined for Eros. The cumulative boulder size distribution per unit area on the entire surface of Itokawa has a power-index of $-3.1 \pm 0.1$ (Fig. 2), which is similar to those on other airless objects. For the east and west sides and the head and body portions of Itokawa, the power-index of the size distributions and the number densities of boulders of these areas are thought to be similar in terms of a statistical point of view. The slope (power-index $-2.8 \pm 0.1$ ) of boulder size distribution on the head is not as steep as the slope (power-index $-3.2 \pm 0.1$ ) of that on the body, and the slope (power-index $-2.9 \pm 0.1$ ) of the size distribution on the west side is not as steep as the slope (power-index $-3.2 \pm$ 0.1 ) of that on the east side. However, the calculation of the rank-sum test indicates that these differences are not statistically significant at the 0.05 probability level. The number density of boulders on the east is roughly the same as that on the west. The number density $\left(1.3 \times 10^{3} / \mathrm{km}^{2}\right)$ on the head is larger than that on the body $\left(8.5 \times 10^{2} / \mathrm{km}^{2}\right)$ by a factor of 1.5 , but the ratio is reduced to 1.3 when only the rough terrains are taken into account for the body. A global mapping of boulders shows that there is no apparent correlation between the locations of boulders and craters. The ratio of the total volume of the boulders ranging from 5 to $40 \mathrm{~m}$ in the mean horizontal dimensions to the total excavated volume of the craters on Itokawa is $~ 25 \%$, which is extremely larger than that on Eros (less than 1\%) and even that on the Moon $(\sim 5 \%)$. These results imply that the boulders on Itokawa cannot solely be produced from the craters.

Based on the appearance of Itokawa's surface and the sizes of the boulders, Fujiwara et al. (2006) and Saito et al. (2006) suggested that such a large number of boulders were produced when Itokawa was generated by a catastrophic disruption. In order to investigate quantitatively whether the numerous boulders were formed only during impact cratering especially when considering the escaping fraction, we calculated the number of boulders and the size of the 
largest boulder using a model based on impact cratering experiments. The estimated number of boulders is smaller than observed data by nearly one order of magnitude, and the calculated size of the largest boulder is smaller than the observed size. These results most likely mean that it is difficult to produce as many boulders on the surface as were observed if only impact cratering is considered as the source; it is likely that most boulders are probably surviving fragments from the parent body of Itokawa, accreted after its breakup, as has been well described in previous papers (Fujiwara et al., 2006; Saito et al., 2006).

Acknowledgments. We would like to thank all members of Hayabusa mission team for their support of the data acquisition. This research was supported by Kobe University through "The 21st Century COE Centers of Excellence Program of the Origin and Evolution of Planetary Systems", and ISAS/JAXA.

\section{References}

Abe, S., T. Mukai, N. Hirata, O. S. Barnouin-Jha, A. F. Cheng, H. Demura, R. W. Gaskell, T. Hashimoto, K. Hiraoka, T. Honda, T. Kubota, M. Matsuoka, T. Mizuno, R. Nakamura, D. Scheeres, and M. Yoshikawa, Mass and local topography measurements of Itokawa by Hayabusa, Science, 312, 1344-1347, 2006.

Britt, D. T., D. Yeomans, K. Housen, and G. Consolmagno, Asteroid density, porosity, and structure, Asteroid III (Univ. Arizona Press), 485-500, 2002.

Cintala, M. J. and K. M. McBride, Block distributions on the lunar surface: A comparison between measurements obtained from surface and orbital photography, NASA Technical Memorandum 104804, NASA, Washington D.C., 1995.

Cintala, M. J., J. B. Garvin, and S. J. Wetzel, The distribution of blocks around a fresh lunar mare crater, Proc. Lunar Planet. Sci. Conf., 13, 100-101, 1982.

Colwell, J. E., Power-law confusion: You say incremental, I say differential, Lunar Planet. Sci. Conf., 24, abstr. 325-326, 1993.

Demura, H., S. Kobayashi, E. Nemoto, N. Matsumoto, M. Furuya, A. Yukishita, N. Muranaka, H. Morita, K. Shirakawa, M. Maruya, H. Ohyama, M. Uo, T. Kubota, T. Hashimoto, J. Kawaguchi, A. Fujiwara, J. Saito, S. Sasaki, H. Miyamoto, and N. Hirata, Pole and global shape of 25143 Itokawa, Science, 312, 1347-1349, 2006.

Dombard, A. J. and A. M. Freed, Thermal stresses on asteroids: application to Eros, American Geophysical Union, Spring Meeting, abstract \#P22B02, 2001.

Fujiwara, A., J. Kawaguchi, D. K. Yeomans, M. Abe, T. Mukai, T. Okada, J. Saito, H. Yano, M. Yoshikawa, D. J. Scheeres, O. Barnouin-Jha, A. F. Cheng, H. Demura, R. W. Gaskell, N. Hirata, H. Ikeda, T. Kominato, H. Miyamoto, A. M. Nakamura, R. Nakamura, S. Sasaki, and K. Uesugi, The rubble-pile asteroid Itokawa as observed by Hayabusa, Science, 312, 1330-1334, 2006

Gaskell, R. W., O. S. Barnoiun-Jha, and D. J. Scheeres, Characterizing small bodies from imaging data, AGU Fall Meeting Abstracts, \#G52B03, 2006.

Geissler, P., J. M. Petit, and R. Greenberg, Ida: Distribution and origin of surface blocks, Lunar Planet. Sci. Conf., 25, abstr. 411-412, 1994.

Geissler, P., J. M. Petit, and R. Greenberg, Ejecta reaccretion on rapidly rotating asteroid: Implications for $243 \mathrm{Ida}$ and 433 Eros, Proc. Astron. Soc. Pacific, 107, 57-67, 1996a.

Geissler, P., J. M. Petit, D. D. Durda, R. Greenberg, W. Bottke, M. Nolan, and J. Moore, Erosion and Ejecta Reaccretion on 243 Ida and Its Moon, Icarus, 120, 140-157, 1996b.

Housen, K. R., Crater ejecta velocities for impact on rocky bodies, Lunar Planet. Sci. Conf., 23, abstr. 555-556, 1992.

Housen, K. R. and K. A. Holsapple, Impact cratering on porous asteroids, Icarus, 163, 102-119, 2003.

Housen, K. R., R. M. Schmidt, and K. A. Holsapple, Crater Ejection Scaling Laws: Fundamental forms based on dimensional analysis, $J$. Geophys. Res., 88, 2485-2499, 1983.

Hirata, N. and A. M. Nakamura, Secondary craters of Tycho: Sizefrequency distributions and estimated fragment size-velocity relationships, J. Geophys. Res., 111, E03005, 2006.

Hirata, N., C. Honda, R. Nakamura, H. Miyamoto, S. Sasaki, H. Demura, A. M. Nakamura, T. Michikami, O. S. Barnouin-Jha, R. W. Gaskell, and J. Saito, Survey of craters and impact structures on the Asteroid Itokawa, Lunar Planet. Sci. Conf., 38, abstr. 1572-1573, 2007.

Lee, S. W., P. C. Thomas, and J. Veverka, Phobos, Deimos, and the Moon: Size and distribution of crater ejecta blocks, Icarus, 68, 77-86, 1986.

Lee, P., J. Veverka, P. C. Thomas, P. Helfenstein, M. J. S. Belton, C. R. Chapman, R. Greeley, R. T. Pappalardo, R. Sullivan, and J. W. Head, Ejecta blocks on 243 Ida and on other asteroids, Icarus, 120, 87-105, 1996.

Melosh, H. J., Impact ejection, spallation, and the origin of meteorites, Icarus, 59, 234-260, 1984.

Michikami, T., Evolution of Asteroid regolith layers by cratering, $\mathrm{PhD}$ Thesis, University of Tokyo, 2001.

Michikami, T., K. Moriguchi, and R. Nakamura, Application to Large Blocks on Asteroid 25143 Itokawa: Ejecta Mass Distribution with Low Velocity for Impact Cratering Experiment on Porous Target, Lunar Planet. Sci. Conf., 36, abstr. 1729-1730, 2005.

Michikami, T., K. Moriguchi, S. Hasegawa, and A. Fujiwara, Ejecta velocity distribution for impact cratering on porous and low strength targets, Planet Space Sci, 55, 70-88, 2007.

Miyamoto, H., H. Yano, D. J. Scheeres, S. Abe, O. Barnouin-Jha, A. F. Cheng, H. Demura, R. W. Gaskell, N. Hirata, M. Ishiguro, T. Michikami, A. M. Nakamura, R. Nakamura, J. Saito, and S. Sasaki, Regolith Migration and Sorting on Asteroid Itokawa, Science, 316, 1011 1014, 2007.

Saito, J., H. Miyamoto, R. Nakamura, M. Ishiguro, T. Michikami, A. M. Nakamura, H. Demura, S. Sasaki, N.Hirata, C. Honda, A. Yamamoto, Y. Yokota, T. Fuse, F. Yoshida, D. J. Tholen, R. W. Gaskell, T. Hashimoto, T. Kubota, Y. Higuchi, T. Nakamura, P. Smith, K. Hiraoka, T. Honda, S. Kobayashi, M. Furuya, N. Matsumoto, E. Nemoto, A. Yukishita, K. Kitazato, B. Dermawan, A. Sogame, J. Terazono, C. Shinohara, and H. Akiyama, Detailed images of asteroid 25143 Itokawa from Hayabusa, Science, 312, 1341-1344, 2006.

Thomas, P. C., J. Veverka, A. Bloom, and T. Duxbury, Grooves on Phobos: Their distribution, morphology, and possible origin, J. Geophys. Res., 84, 8457-8477, 1979.

Thomas, P. C., J. Veverka, R. Sullivan, D. P. Simonelli, M. C. Malin, M. Caplinger, W. K. Hartmann, and P. B. James, Phobos: regolith and ejecta blocks investigated with Mars Orbiter Camera images, J. Geophys. Res., 105, 15091-15106, 2000.

Thomas, P. C., J. Veverka, M. S. Robinson, and S. Murchie, Shoemaker crater as the source of most ejecta blocks on the asteroid 433 Eros, Nature, 413, 394-396, 2001.

T. Michikami (e-mail: mitikami@fukushima-nct.ac.jp), A. M. Nakamura, N. Hirata, R. W. Gaskell, R. Nakamura, T. Honda, C. Honda, K. Hiraoka, J. Saito, H. Demura, M. Ishiguro, and H. Miyamoto 